NBER WORKING PAPER SERIES

\title{
REMIX RIGHTS AND NEGOTIATIONS OVER THE USE OF COPY-PROTECTED WORKS
}

\author{
Joshua S. Gans
}

Working Paper 20364

http://www.nber.org/papers/w20364

\author{
NATIONAL BUREAU OF ECONOMIC RESEARCH \\ 1050 Massachusetts Avenue \\ Cambridge, MA 02138 \\ August 2014
}

I thank Ariel Katz, Shane Greenstein, Avi Goldfarb, an anonymous referee, and seminar participants at the University of Toronto Law School for helpful comments. The views expressed herein are those of the author and do not necessarily reflect the views of the National Bureau of Economic Research.

The author has disclosed a financial relationship of potential relevance for this research. Further information is available online at http://www.nber.org/papers/w20364.ack

NBER working papers are circulated for discussion and comment purposes. They have not been peerreviewed or been subject to the review by the NBER Board of Directors that accompanies official NBER publications.

(C) 2014 by Joshua S. Gans. All rights reserved. Short sections of text, not to exceed two paragraphs, may be quoted without explicit permission provided that full credit, including $(\mathbb{C}$ notice, is given to the source. 
Remix Rights and Negotiations Over the Use of Copy-Protected Works

Joshua S. Gans

NBER Working Paper No. 20364

August 2014, Revised June 2015

JEL No. O34

\begin{abstract}
$\underline{\text { ABSTRACT }}$
This paper examines an environment where original content can be remixed by follow-on creators. The modelling innovation is to assume that original content creators and remixers can negotiate over the 'amount' of original content that is used by the follow-on creator in the shadow of various rights regimes. The following results are demonstrated. First, traditional copyright protection where the original content creators can block any use of their content provides more incentives for content creators and also more remixing than no copyright protection. This is because that regime incentivises original content creators to consider the value of remixing and permit it in negotiations. Second, fair use can improve on traditional copyright protection in some instances by mitigating potential hold-up of follow-on creators by original content providers. Finally, remix rights can significantly avoid the need for any negotiations over use by granting those rights to follow-on innovators in return for a set compensation regime. However, while these rights are sometimes optimal when the returns to remixing are relatively low, standard copyright protection can afford more opportunities to engage in remixing when remixing returns are relatively high.
\end{abstract}

Joshua S. Gans

Rotman School of Management

University of Toronto

105 St. George Street

Toronto ON M5S 3E6

and NBER

joshua.gans@gmail.com 


\section{Introduction}

Remixing is a term used to describe taking content (sound, music, photos and words) and altering them in some manner to create new content. While the notion of derivative works has existed in copyright law for some time, digital technology has allowed a wider range of content types to be combined to produce remixed creative works and for that work to reach a wider audience. For example, users now attract millions of views on YouTube with their own video representations of popular songs (e.g., the Harlem Shake phenomenon) or re-working of television shows and movies (e.g., the synchronisation of video of George W. Bush and Tony Blair to the duet, Endless Love). For the purposes of this paper, remixing occurs whenever someone takes copy protected content and repurposes it in some manner. Thus, while it does not include direct copying, parts, or maybe all, of the copy protected material are used in the derivative work.

Legal and economic scholars have been challenged in considering how such remixing should be treated. In law, there is a position that it may fall under "fair use." ${ }^{2}$ Fair use is an exemption to copyright protection, that exists in some jurisdictions, related to works that use copy protected materials for reviews, discussion and parody. This may, in some circumstances, encompass remixed content. However, it should be noted that a fair use exemption does not exist everywhere; including, notably, Europe and Australia. Moreover, while fair use can potentially be readily applied to noncommercial remixing and use of copy-protected material, when it is uploaded or distributed using commercial platforms (such as YouTube or SoundCloud), the precise nature of the content becomes ambiguous.

In this paper, we explore remix rights in the context of considering the optimality of copyright law from a property rights perspective. That is, the law sets default rights on various parties. Usually, in fact, no copy-protected material can be used in any form by others unless express permission is given by the copyright owner. Thus, potential user/creators need to obtain permission to use such materials. As Lessig (2008) notes, this has occurred on open platforms such as YouTube where amateurs, for instance, uploaded videos with songs playing in the background only to be cited with takedown notices because appropriate permissions had not been sought. These takedown notices invariably occurred after remix effort had been expended. In other cases cited by Lessig, users who sought permissions found transaction costs prohibitive. Google and other platforms have since opted for solutions that encourage remixed content and lower transaction costs for obtaining permissions.

\footnotetext{
${ }^{2}$ For a review see Katz (2013).
} 
Lessig (2008) argues that these measures still stifle creativity. He claimed that technology has made copying so easy that it is hard to base the structure of the law on the presumption that copying can be prevented. He argues that remix rights should be applicable where the user has a demonstrably amateur characteristic. For instance, a song in the background of a family video is not copy-protected but if it is uploaded to YouTube and publicly available it becomes so. Indeed, in Canada, new provisions on user-generated content reflect this. These provisions allow remixing so long as the purpose is non-commercial in nature and does not have a substantial adverse effect on the copyright holder. However, it should be noted that remixing involves activities that may not satisfy these criteria. This has prompted some to propose alternative means of compensating creators while allowing pervasive copying. For instance, the European-based Right2Remix.org argues that remix rights should be granted by default as they are a form of creative expression with lump-sum compensation being paid to the copyright owners for any losses incurred as a result of this.

In economics, Boldrin and Levine (2010) argue for a permissive regime that essentially removes copy-protection, claiming that incentives for creators will be adequate in their absence. Lessig also argues that the regulation of small scale technical copyright infringement does not outweigh the legal and transactional costs involved. This is a pervasive theme. When economists looked at the desirability of a fair use exemption they emphasised the transaction costs involved in securing permissions for derivative works (Landes and Posner, 1989), in particular, when those works involve combinations of work by diverse copyright holders (Depoorter and Parisi, 2002). However, to date, economists have not considered copyright protection from a perspective where the costs of transacting come from potential hold-up -- either by content creators (when copyright protection is strong) or users who remix (when copyright protection is weak). ${ }^{3}$ That is the contribution of this paper.

This paper examines the impact of remixing and the various rights regimes that have been proposed. As a benchmark, we consider how different regimes impact on the overall quality of creative works generated -- both original content and remixing -- and also on whether a regime can leave incentives to create original content no lower than what would arise it remixing were technically infeasible.

The modelling innovation is to move beyond the simple view of copyright protection as protecting the original content creator from competition from copies (see Novos and Waldman,

\footnotetext{
${ }^{3}$ Halonen-Akatwijuka and Regner (2009) consider the optimal ownership of copyright itself between music artists and publishers/distributors. However, they do not examine the optimal copyright law as is done here.
} 
1984; Johnson, 1985; and Landes and Posner, 1989, for a classic treatments). Instead, as with the incomplete contracts literature in the theory of the firm (Hart, 1995) and also its application to law (Pitchford and Snyder, 2003), I consider, carefully, how different rights regimes specifying residual control and return rights impact on negotiations between original content creators and users who remix for public consumption. In effect, I bring the cumulative innovation framework beyond patent matters (Green and Scotchmer, 1995) to copyright matters. This not only allows us to examine remix rights and their impact but also to consider more carefully the impact of traditional copyright protection as it currently exists on the amount of remixing that occurs. As a consequence, it explicitly considers hold-up as a transaction cost in the copyright area. ${ }^{4}$

The outline of the paper is as follows. In section II, I introduce a baseline model involving the interactions between an initial content creator and a follow-on remixer of that content. These agents undertake non-contractible investments in the quality of their (potentially) published works and then negotiate in the shadow of copyright regimes that might exist. In order to focus on the interesting case, the model makes the assumption that, in the absence of permission remixing harms the copyright owner, although, in reality, it is possible that, in some instances, the owner may receive benefits from remixing. Section III considers the outcomes when there are traditional rights regimes of either no copyright protection or traditional copyright protection. In the former, followon remixers have a right to remix and publish their work while in the latter, initial content creators have a right to block the publication of remixed work. In each case, the rights determine the bargaining position of each agent in negotiations over whether remixed work is actually published or not. The significant result is that traditional copyright protection enhances the incentives of initial content creators to invest in their content -- something that is intuitive -- but also the actual amount of remixing that occurs and is published -- a result that is counter-intuitive at first glance. That latter result comes from the incentives of initial content creators in the absence of copyright protections to lower their own quality enough to discourage remixing from taking place and cannabilising their own returns. Under copyright protection, there is no such restraint and so socially superior outcomes -- in terms of the welfare of initial content providers and follow-on remixers -- arise.

Section IV looks at 'fair use' exemptions. While in many legal studies these have been envisaged as allowing copy protected work to be used for specific purposes (e.g., parody), here I consider fair use as a safe harbour based on the 'quantity' of a copy protected work that is used. For

\footnotetext{
${ }^{4}$ A related paper by Miceli and Adelstein (2006) looks at fair use exemptions as involving a minimum standard of copying by horizontally differentiated competitors to the copyright holder. It derives a socially optimal fair use standard under those assumptions but does not consider the underlying bargaining game as I do here.
} 
example, artists may be able to lift samples of music and incorporate them without permission or payment but not entire pieces of content. Here, it is found that fair use only binds when some remixing will be desired in equilibrium and will often involve additional payments to copyright owners. This reinforces the finding that no copyright protection is sub-optimal but also the notion that if the share of original content returns is heavily dependent on a lack of sampling, then it is socially optimal not to have a fair use exemption. This mirrors, in part, the notion that fair use should be accompanied by non-commercial follow-on use.

Section $\mathrm{V}$ then considers remix rights as outlined by some advocacy groups. Such rights change the defaults of blocking by copyright holders to allow remixers to engage in such activities but also being under an obligation to compensate copyright holders for potential losses. It is demonstrated that such a regime can encourage second best investments in remixed content without reducing initial content investment beyond that would arise if remixing was blocked. Moreover, remix rights avoid the need for negotiations between the original content provider and the remixer prior to remixing and, perhaps, altogether. In other words, remix rights can minimize transaction costs. That said, because traditional copyright protection can lead to actual remixing, it is not always the case that remix rights generate a higher level of remixed content being created and published. Overall, from a welfare perspective, it is not possible to clearly rank remix rights over traditional copyright protection. In an extension, a remix regime similar to that currently provided by YouTube is examined which has some superior elements but also some mitigating effects compared with other copyright regimes considered.

\section{Baseline Model}

We model a content creator, $A$, and a user/remixer, $B$. There are two time periods (Figure 1). In period 1, a copyright regime is established which is known to all. Also in that period, $A$ creates content by expending effort (or other resources) of measure, $x$. The cost of $x$ is $C(x)$ which is increasing and convex. In period 2, $B$ takes $A$ 's content and chooses whether to remix it. As a simple measure, suppose that $s$ is the share of $A$ 's content that $B$ uses in remixing. ( $s$ could stand for 'sample'). In addition, $B$ expends their own effort, $y$, in taking $s$ and transforming it into their own content. Again, the cost of $y$ is $C(y)$ which is increasing and convex. Once $B$ has made their choice, $A$ and $B$ bargain over $s$ that can be ultimately released to others as $B$ 's content and any payment $(t)$ from $A$ to $B$ in return for this. The outcome of that bargain is then implemented and payoffs are realized. The timing of this game mirrors a standard incomplete contracts framework where $x$ and $y$ 
are non-contractible (that is, observable but not verifiable choices) while $s$ and payments are contractible.

\section{A note on timing}

Of course, a key assumption here is that $A$ and $B$ cannot negotiate prior to $A$ creating their content. There are a couple of reasons for this. As noted already, $x$ is reasonably assumed to be noncontractible in many creative settings. Second, $A$ may not be aware of the " $B$ 's" out there prior to creating that content and so could not negotiate with them (see Pitchford and Snyder, 2003). Either way, this prevents an ex ante deal that would otherwise generate an optimal outcome regardless of the regime in place. Third, negotiation over $s$ takes place after $y$ has been chosen. The reason for this is that, as $y$ is non-contractible, even if $s$ is agreed upon, once $y$ is realized and observed there is an incentive to renegotiate $s$.

The timing assumption that $s$ is negotiated after $y$ has been chosen is worthy of additional discussion. As is familiar from the literature on property rights (Hart, 1995), when a key variable, in this case $y$, is non-contractible, this means that even signed formal contracts over other variables will be subject to renegotiation. A formal contract's terms can be amended if both parties to the contract agree. Thus, suppose that, for example, $A$ and $B$ agree, prior to $y$ being chosen, that $B$ can sample $s_{c}$. They also agree on a payment, $t_{c}$, from $B$ to $A$ for that right. $B$ then chooses a $y$ such that if the chosen sample were greater than $s_{c}$, its own payoff would be higher. Consequently, $B$ may offer $A$ an amount greater than $t_{c}$, for that additional sample. $A$ could refuse but it is also conceivable that $A$ may prefer the higher compensation over any perceived losses from the increment to the sample used by $B$. This, of course, makes modelling the outcomes of the game a challenge. How property rights theory resolved this issue, was to leave the negotiation of variables like $s$ and $t$ until after $y$ is chosen. In this model, $A$ and $B$ would form rational expectations regarding whether an agreement might be reached ex post and also the terms of that agreement. The theory would then look for an equilibrium where those expectations were met. This is the approach we follow here. So while $s$ and $t$, in reality, can be negotiated prior or after $y$ being chosen, the model's outcome based on ex post negotiation of these terms generates the same equilibrium prediction.

\section{Payoffs and bargaining}

The payoffs to each agent are as follows. It is assumed that the payoff $A$ receives exclusive of creation costs is $\pi(x, s)+t$ which is assumed to be increasing and concave in $x$ and non-increasing 
in $s$ with $\pi(0, s)=0$. B's payoff is $u(x, y, s)-C(y)-t$ which is assumed to be increasing, concave and supermodular in $(x, y, s)$. We assume that $u(0, y, s)=u(x, y, 0)=0$ (that is, content from $A$ and access to original content is required for $B$ to earn a positive payoff).

Below I will often rely on the following functional form assumptions -- consistent with the above assumptions -- to derive more precise predictions and show the possibility of various outcomes. Specifically I will assume that $C(x)=\frac{1}{2} x^{2}, C(y)=\frac{1}{2} y^{2}, \pi(x, s)=\beta x+x(1-s)$ and $u(x, y, s)=\theta x y s$. The latter assumptions are of particular interest as they allow for the possibility that each 'unit' of content remixed reduces the direct revenue to $A$; indeed, for $s=1, \pi=\beta x$; the revenue independent of sampling. Consequently, it allows for the more dire predictions from copyright advocacy groups (especially if $\beta=0$ ). In addition, the parameter, $\theta$, is, in many respects, a proxy for the remix 'opportunity.' This will be useful in considering the impact of digitisation on the optimal copyright regime as it may be associated with a general rise in $\theta$.

However, it should be emphasised that much remixed content does not involve $s=1$. Instead, it may involve some amount less than 1 with anything beyond that amount not increasing $B$ 's utility. In that respect, one should consider $s=1$ as a normalised amount to the maximum $B$ could possibly value. This interpretation is not innocuous, however, as in the specific functional form, it may be enough to remove $A$ 's direct profits from their content; although it will still receive a remixindependent amount of $\beta$.

In negotiations over $(s, t)$, it is assumed that $A$ and $B$ use the Nash bargaining solution assigning asymmetric bargaining power to $A$ of $\alpha \in(0,1)$. This solution implies that:

$$
\begin{gathered}
s^{*} \in \arg \max _{s} \pi(x, s)+u(x, y, s) \\
t^{*}=\alpha\left(u\left(x, y, s^{*}\right)-o_{B}(x, y)\right)-(1-\alpha)\left(\pi\left(x, s^{*}\right)-o_{A}(x, y)\right)
\end{gathered}
$$

where $o_{A}$ and $o_{B}$ are $A$ and $B$ 's respective outside options. Anticipating this, $A$ and $B$ will set their effort levels according to:

$$
\begin{gathered}
x^{*} \in \arg \max _{x} \alpha\left(\pi\left(x, s^{*}\right)+u\left(x, y^{*}(x), s^{*}\right)-o_{B}\left(x, y^{*}(x)\right)\right)+(1-\alpha) o_{A}\left(x, y^{*}(x)\right)-C(x) \\
y^{*}\left(x^{*}\right) \in \arg \max _{y}(1-\alpha)\left(\pi\left(x^{*}, s^{*}\right)+u\left(x^{*}, y, s^{*}\right)-o_{A}\left(x^{*}, y\right)\right)+\alpha o_{B}\left(x^{*}, y\right)-C(y)
\end{gathered}
$$

Thus, each agent internalizes a fraction of the surplus generated $(\pi+u)$ but also takes into account the impact of their effort on the various outside options. In addition, $A$ acts as a Stackelberg leader when expending effort and takes into account the impact of their decision on $B$ 's choices. Importantly, as the negotiated $s$ only enters into the joint surplus term, by the envelope theorem, 
both $A$ and $B$ take it as given when choosing their own efforts. To see this, observe that $A$ and $B$ 's first order conditions are:

$$
\begin{gathered}
\alpha\left(\frac{\partial \pi}{\partial x}+\frac{\partial \pi}{\partial s}\left(\frac{d s^{*}}{d x}+\frac{d s^{*}}{d x} \frac{d y^{*}}{d x}\right)+\frac{\partial u}{\partial x}+\frac{\partial u}{\partial y} \frac{d y^{*}}{d x}+\frac{\partial u}{\partial s}\left(\frac{d s^{*}}{d x}+\frac{d s^{*}}{d x} \frac{d y^{*}}{d x}\right)-\frac{\partial o_{B}}{\partial x}-\frac{\partial o_{B}}{\partial y} \frac{d y^{*}}{d x}\right)+(1-\alpha)\left(\frac{\partial o_{A}}{\partial x}+\frac{\partial o_{A}}{\partial y} \frac{d y^{*}}{d x}\right) \leq \frac{\partial C}{\partial x} \\
(1-\alpha)\left(\frac{\partial \pi}{\partial y}+\frac{\partial \pi}{\partial s} \frac{d s^{*}}{d y}+\frac{\partial u}{\partial y}+\frac{\partial u}{\partial s} \frac{d s^{*}}{d y}-\frac{\partial o_{A}}{\partial y}\right)+\alpha \frac{\partial o_{B}}{\partial y} \leq \frac{\partial C}{\partial y} C(y)
\end{gathered}
$$

Note that the first order condition for $s$ in the Nash bargaining solution implies that $\frac{\partial \pi}{\partial s}+\frac{\partial u}{\partial s}=0$, thus, these first order conditions simplify to:

$$
\begin{gathered}
\alpha\left(\frac{\partial \pi}{\partial x}+\frac{\partial u}{\partial x}+\frac{\partial u}{\partial y} \frac{d y^{*}}{d x}-\frac{\partial o_{B}}{\partial x}-\frac{\partial o_{B}}{\partial y} \frac{d y^{*}}{d x}\right)+(1-\alpha)\left(\frac{\partial o_{A}}{\partial x}+\frac{\partial o_{A}}{\partial y} \frac{d y^{*}}{d x}\right) \leq \frac{\partial C}{\partial x} \\
(1-\alpha)\left(\frac{\partial \pi}{\partial y}+\frac{\partial u}{\partial y}-\frac{\partial o_{A}}{\partial y}\right)+\alpha \frac{\partial o_{B}}{\partial y} \leq \frac{\partial C}{\partial y} C(y)
\end{gathered}
$$

As those actions do impact on the realised $s^{*}$, we will look for a fixed point outcome (i.e., rational expectations equilibrium) in what follows.

\section{Traditional Rights Regimes}

I now examine the two traditional rights regimes. First, where there is no copyright protection. Second, where there is copyright protection that gives control over copying to the content creator (consistent with current law).

\section{No copyright protection (NC)}

As a first step, consider what happens when there is no copyright protection. In this case, absent an agreement, $B$ would be free to remix as much of $A$ 's content as they would like. As $u$ is increasing in $s$, this would lead to a choice of $s_{N C}=1$. Thus, in this situation, $o_{A}=\pi(x, 1)$ and $o_{B}=u(x, y, 1)$.

The immediate consequence of this is that the negotiated $t$ must be negative if $s_{N C}<1$ :

$$
t_{N C}=\alpha \underbrace{\left(u\left(x, y, s_{N P}\right)-u(x, y, 1)\right)}_{<0}-(1-\alpha) \underbrace{\left(\pi\left(x, s_{N C}\right)-\pi(x, 1)\right)}_{>0}
$$

so that $A$ is paying $B$ in return for it not using its whole content in a remix. Of course, this depends on the negotiated $s$ actually being strictly less than 1 . The following proposition summarizes the possible equilibrium outcomes.

Proposition 1. If $\frac{\partial \pi(0,1)}{\partial x}>C^{\prime}(0)$, then, in equilibrium, $x_{N C}>0$. If $\frac{\partial \pi(0,1)}{\partial x} \leq C^{\prime}(0)$, then a necessary condition for an equilibrium with $s_{N C}<1$ and $x_{N C}>0$ to exist is that there is an $x$ and $s<1$ such that $\pi(x, s)+u\left(x, y^{*}(x, s), s\right)>\pi(x, 1)+u\left(x, y^{*}(x, 1), 1\right)$ and $\pi(x, s) \geq C(x)$. 
The intuition for this result is as follows. Clearly, if there is a positive net marginal return to $x$ even if $s=1$, then a positive investment by $A$ will occur in equilibrium. On the other hand, if this isn't the case, then $A$ will choose effort that, if feasible, will cause a negotiated $s$ less than 1 . Specifically, it will choose $x$ such that with $B$ 's response to that $x$, the joint surplus weighing $A$ 's revenues is higher than those weighing $B$ 's. As $x$ and $y$ are complements for $B$, this will be a relatively low value of $x$. If $A$ chose an $x$ that was too high, then in negotiations, $B$ will insist upon a level of $s=1$ and $A$ would earn a negative return. Consequently, under NC, $A$ restrains its investment in content to ensure the content is not too attractive to $B$.

To see this more clearly consider what happens under the specific functional form assumptions stated above. First, if $s$ is negotiated:

$$
s_{N C}=\left\{\begin{array}{ccc}
1 & & y \theta>1 \\
0 & \text { if } & y \theta \leq 1
\end{array}\right.
$$

Working backwards $y_{N C}=\theta x((1-\alpha) s+\alpha)$. A takes this into account when choosing $x$, resulting in:

$$
x_{N C}=\frac{\beta+\alpha(1-s)}{1+2(1-s) \alpha(s(1-\alpha)+\alpha) \theta^{2}}
$$

Note that $x_{N C}$ is decreasing in $B$ 's value (as parameterized by $\theta$ ); that is, the choice of $x$ by $A$ is a strategic substitute with $y$. Given this, the equilibrium outcomes are characterized by the following proposition.

Proposition 2. If $\beta>\frac{1}{\theta^{2} \alpha}+2-\alpha$, then $s_{N C}=1, x_{N C}=\beta$ and $y_{N C}=\theta \beta$. Otherwise, $s_{N C}=0$, $x_{N C}=\frac{\beta+\alpha}{1+2 \alpha^{2} \theta^{2}}$ and $y_{N C}=\frac{(\beta+\alpha) \alpha \theta}{1+2 \alpha^{2} \theta^{2}}$.

The proof of the proposition is in the appendix. It is instructive to note that, consistent with Proposition 1, if $\beta=0$, then $s_{N C}=0$ and no remixed content is published. However, even when $\beta>0$ is positive the negotiated $s_{N C}$ can equal 0 while $y_{N C}>0$. So remixing, as a creative activity, takes place and, moreover, an improvement in A's bargaining position increases that activity. However, from a welfare perspective, $B$ 's investment is purely wasteful and is only made to improve their bargaining position in negotiations with $A$. A's bargaining power does not play a direct role as the regime shifts to one where $B$ can use all of $A$ 's material. However, that regime is more likely to arise when $A$ has more bargaining power as it appropriates more of the value $B$ creates. 
At a first glance, this result reaffirms a standard approach to copyright in that, in the absence of protection, content creators will only have an incentive to create if they would not be harmed too much by remixing. However, regardless, it demonstrates that a content creator will take into account the impact their creation has on incentives to remix and hence, the amount they might have to pay to prevent such remixing from occurring. Because content creators can create with an eye to how their works might be remixed, in the absence of copyright protection, they have an incentive to produce works that are hard to remix. There are many ways this might manifest themselves. For instance, Landes and Posner (1989) argue that writers would hold off releasing content until they themselves had produced a translation to other languages. The point here being is that the absence of copyright protection does not so much diminish the incentives to create content but the type of content that is created and the room it offers others to build upon.

\section{Copyright protection $(\mathrm{CP})$}

Standard copyright protection gives the content creator the right to prevent any copying of their material. In the notation expressed here, $A$ can set $s$ and, in the absence of an alternative agreement, will set $s=0$. Consequently, in negotiations over $(s, t), o_{B}=0$ while $o_{A}=\pi(x, 0)$.

Given the general form of payoff functions here, it is difficult to characterize how copyright protection changes the equilibrium choices of $A$ and $B$. A first insight is that while no copyright

protection ruled out $s=1$ in equilibrium, it is possible that $s_{C P}=1$ even if $\frac{\partial \pi(0,1)}{\partial x} \leq C^{\prime}(0)$. This is because, under $\mathrm{CP}$, the equilibrium payment from $B$ to $A, t$, is positive. Thus, $A$ shares in $u(x, y, s)$ whereas under NC, $A^{\prime}$ 's interests are in minimizing $u(x, y, 1)-u(x, y, s)$ which because $s$ and $y$ are complements, causes $A$ to choose an $x$ that ensures $y$ is low.

Second, the direct impact of moving from NC to CP on agent's incentives are exactly what one would expect. Holding $y$ fixed, the marginal return to $x$ for $A$ increases under CP. Similarly, holding $x$ fixed, $B$ 's marginal return to $y$ decreases under CP. However, because these impacts conflict, when $A$ and $B$ 's efforts are strategic complements, we cannot say, in equilibrium, what the overall impact of a move from $\mathrm{NC}$ to $\mathrm{CP}$ is. Nonetheless, it is possible that $y$ under $\mathrm{NC}$ is a strategic substitute for $A$. In this case, $x$ will be higher in equilibrium under CP than under NC.

Given this ambiguity, it is useful to return to specific functional forms. Note that the negotiated $s$ will be determined by the same formula as this is an ex post efficient choice, however, the precise value will depend on the value of $y$ realized. In this case, $y_{C P}=\theta x(1-\alpha) s$ while 
$x_{C P}=\frac{1+\beta-s \alpha}{1-2 s^{2} \alpha(1-\alpha) \theta^{2}}$ which is positive so long as A's objective function is concave. Note that, unlike the case for $\mathrm{NC}$, here, an increase in $B$ 's value (as parameterized by $\theta$ ) increases $x$ in equilibrium.

The following proposition compares the equilibrium outcomes under $\mathrm{CP}$ to those under NC.

Proposition 3. The threshold level of $\beta$ (i.e., $\beta>\beta_{C P}=\frac{1}{\theta^{2}(1-\alpha)}-1-\alpha$ ) that gives rise to remixed content being published in equilibrium under CP is lower than under NP. For any parameters, $x_{C P}>x_{N C}$, while, $y_{C P}>y_{N C}$ when $s_{C P}=1$.

Once again, the proof is in the appendix. $x_{C P}>x_{N C}$ always (even for a range of parameters whereby $s_{C P}=1$ and $\left.s_{N C}=0\right)$ but when $s_{C P}=0, y_{C P}=0$ and is, therefore, less than $y_{N C}$. However, this remix is not published (rendering any positive $y$ socially inefficient) and thus, $\pi+u$ is always higher under CP than under NC. Intuitively, under CP, $A$ appropriates a greater return from their own effort (as per standard theories of copyright protection) but, in addition, the negotiated $s$ is more likely to be permissive to follow-on remixing precisely when such activities are highly valuable socially.

This demonstrates there is considerably more subtlety that operates with copyright regimes than is commonly expressed. In particular, this model demonstrates that, in an environment where remixing can be very damaging to the profits of the content creator, the assignment of copyright protection to that creator can result in more remixing than when such rights are not present. The reason for this is that assigning copy protection rights to the content creator allows that creator to appropriate some of the returns from remixing. Consequently, they are more open to such remixing even at the sacrifice of profits from the original work. This demonstrates that a potential unintended consequence of removing copyright protection in order to remove barriers to remixing is, in fact, to create conditions whereby remixing does not occur.

\section{Fair Use Regime}

This section turns to consider fair use. Here fair use is not interpreted with respect to the precise use the copyrighted work is put to but to the extent of that use. This gives us some sense as to the optimality of providing a regime that is somewhat intermediate to the two traditional regimes considered thusfar.

Following Miceli and Adelstein (2006), here, fair use is modelled as a minimum 'amount,' $\underline{s}$, set by the policy-maker, that $B$ can use without $A$ 's permission. Thus, should $A$ and $B$ fail to reach an agreement otherwise, $A$ will receive $\pi(x, \underline{s})$ while $B$ will receive $u(x, y, \underline{s})$. Clearly, from this perspective, fair use is a regime weakening copyright protection from a full copy protected regime but is stronger than no copy protection. 
The following proposition characterizes the optimally chosen fair use regime for our specific functional form.

Proposition 4. For $\beta$ sufficiently low, fair use is not optimal. For $\beta$ sufficiently high, fair use is optimal (i.e., the policy-maker will set $\underline{s}>0$ ) but never involves $\underline{s}=1$.

This result is an extension of Proposition 3 that found that CP generated higher surplus than NP. However, it also demonstrates that $\mathrm{CP}$ may not be optimal when the share of $A$ 's profits that varies with use of the work by $B$ is low. In this case, providing $B$ with some rights improves their bargaining position and leads to higher surplus being generated part of which is redistributed to $A$ as payment for a negotiated $s>\underline{s}$. Specifically, whenever fair use is optimal, it is also efficient to negotiate $s=1$. Thus, in this situation, fair use is a mechanism that allows $A$ to appropriate some share of $B$ 's payoff, and thus gives them an incentive to improve content quality.

An interesting aspect of the equilibrium outcome here is that fair use is never used as a weapon on the optimal regime. Of course, the model here considers the regime dependent upon the particular characteristics of $A$ and $B$. More generally, optimal fair use should apply regardless of those characteristics. This might generate a concern that a fair use option could be used by potential follow-on content users to threaten the destruction of original content holder rents. However, here the optimal regime should not involve that threat and, if there are negotiations, it would be to extend use beyond fair use and certainly not involve any payments from $A$ to $B$. This suggests that there is some rationale for the notion that fair use can apply subject to a 'harm to the original content provider' test; the idea being that $A$ would be able to prevent remixing if harmed, thus, endogenously setting their bargaining positioning while allowing remixing to occur in cases where there is no harm to $A$.

\section{Remix Rights with Compensation}

We now consider an alternative rights regime. Whereas under both $\mathrm{NC}$ and $\mathrm{CP}$, a particular agent is given the right to choose $s$ in the absence of an agreement here that right is de-coupled from a right to receive compensation for that choice.

The Remix Right regime proposed by Right2Remix.org involves granting all users:

- Transformative usage rights: "the right to change works during usage and publish the results" (e.g., background music in mobile phone videos);

- Remix rights: "the right to create and publish remixes of existing works" (e.g., a fake trailer for a TV series); 
- Remix Commercialization rights: "the right to commercialize remixes" (e.g., selling music mash-ups on iTunes);

-... in exchange for lump-sum compensation (or appropriate compensation for commercialization)

In the language of the model presented thusfar, this amounts to giving $\boldsymbol{B}$ the right to choose $\boldsymbol{s}$ but if $\boldsymbol{B}$ exercises that right, $\boldsymbol{B}$ must pay $\boldsymbol{A}$ compensation. This leaves open the precise form of the compensation and also the amount. We will consider some variants in what follows. We note here that these are proposed rights rules that we explore in order to determine whether giving $B$ the right to remix without permission from $A$ may be superior to other rights regimes already considered above.

\section{Lump-Sum Compensation (RR)}

A natural contender for lump-sum compensation that is potentially measurable is compensation equal to the amount $A$ would have expected to receive if $A$ had unilaterally made their preferred choice with regard to remix rights. Given the assumptions stated thusfar, $A$ 's preferred choice is to set $s=0$. In this case, if $B$ set $s, B$ would have to pay $A \pi(x, 0)-\pi(x, s)$.

In this situation, given $x$ and $y, B$ will choose $s$ to solve:

$$
\max _{s} u(x, y, s)+\pi(x, s)-\pi(x, 0)
$$

Thus, $s$ will be set to maximise joint surplus (as it would in the negotiations considered in the previous section). Here, however, $B$ 's payoff is as if bargaining power, $\alpha=0$, under a CP regime. Note that because of this and because negotiations are otherwise ex post efficient, $B$ has no incentive to negotiate an alternative arrangement with $A$. Consequently, there are no transaction costs associated with bargaining over $s$ because no bargaining over $s$ takes place. $B$ implements the efficient outcome unilaterally. Thus, the significance of a remix rights regime is precisely that it obviates the need for ex post negotiations over the use of A's content.

Given this, $B$ chooses $y$ to solve:

$$
\max _{y} u\left(x, y, s^{*}\right)+\pi\left(x, s^{*}\right)-\pi(x, 0)-C(y)
$$

which yields a value of $y$ consistent with the first best holding $x$ fixed. For $A$, it receives $\pi(x, 0)$ regardless of whether $B$ chooses $s$ or leaves the choice to $A$. Thus, it solves:

$$
\max _{x} \pi(x, 0)-C(x)
$$

This is not consistent with the first best as it fails to take into account any positive externality on $B$. However, compared to a copyright protection regime without negotiation, $A$ is no worse off. 
While a remix rights regime appears to have some favourable efficiency properties, it is important to compare it more closely with conventional copyright regimes. The following proposition characterises that comparison for the specific functional forms assumed above where $\mathrm{RR}$ denotes the remix rights regime.

Proposition 5. For $\theta$ and $\beta$ sufficiently low (i.e, $\theta<\frac{1}{\sqrt{(1-\alpha)(\beta+1+\alpha)}}$ ), RR is (weakly) optimal. Specifically, if $\theta<\min \left[\frac{1}{\sqrt{(1-\alpha)(\beta+1+\alpha)}}, 1\right]$, the equilibrium outcomes under RR and CP are identical; if $\theta \geq \max \left[\frac{1}{\sqrt{(1-\alpha)(\beta+1+\alpha)}}, 1\right], \quad s_{R R}=s_{C P}=1, \quad x_{C P}>x_{R R}$ and $y_{C P}>y_{R R}$; if $\frac{1}{\sqrt{(1-\alpha)(\beta+1+\alpha)}}>\theta \geq 1$ (which can arise if $\left.\beta \leq \frac{\alpha^{2}}{1-\alpha}\right), \quad s_{R R}=1>s_{C P}=0, \quad x_{R R}=x_{C P}>x_{N C}$ and $y_{R R}>y_{C P}=0$; and if $1>\theta \geq \frac{1}{\sqrt{(1-\alpha)(\beta+1+\alpha)}}$ (which can arise if $\beta>\frac{\alpha^{2}}{1-\alpha}$ ), $s_{C P}=1>s_{R R}=0, x_{C P}>x_{R R}$ and $y_{C P}>y_{R R}=0$.

The significance of this result is that remix rights allow remixing to occur without negotiation over a permission to remix. Figure 2 depicts the optimal outcomes. Moreover, it is demonstrated that, in some situations, in particular, where $\beta$ and $\theta$ are high, $\mathrm{CP}$ will, in fact, be more permissive of remixing than RR. This is because in those cases, the returns to both original content provision and remixing are sufficiently high that $A$ has an incentive, ex post, to negotiate for high levels of remixing.

Proposition 5 demonstrates that there is no simple comparison between RR and CP. Nonetheless, it is never the case that $A$ 's effort under RR exceeds its effort under CP. This is because under RR, A's effort is based solely on a situation that would arise if remixing were not possible. There are times, however, where total value created is higher under RR than under CP (if the former involves remixing while the latter does not) as $B$ 's incentives are efficient conditional on $x$. However, in other cases, total value created is higher in this case under CP than RR.

What is driving this outcome is that $\mathrm{CP}$ gives $A$ a stake in the value generated by remixing whereas RR does not. As $A$ 's effort increases that value, there is a direct incentive to increase $x$ precisely when remixing is more valuable. In this particular case, despite the fact that $B$ 's incentives to choose $y$ under $\mathrm{CP}$ are not, ceteris paribus, as strong as its incentives under RR, $y$ is higher because it is complementary with $x$. It could, of course, under an alternative specification for the value created by original content and remixing have been the case that $y$ under RR was higher than $y$ under $\mathrm{CP}$ and, indeed, because of complementarity, $x$ may also be lower. Nonetheless, by construction, under $\mathrm{CP}$, both $A$ and $B$ potentially can have an interest in increasing the value realized under remixing whereas under RR only $B$ has that incentive while $A$ is ambivalent. 


\section{Revenue Sharing Compensation ( $R C)$}

The lump-sum compensation approach suggests that $B$ might have an interest in allowing $A$ to share some of their remix value (or revenue) in order to increase effort expended on $x$ beyond that focussed on original content revenue alone. Given this, here I consider an alternative approach to remix rights and compensation based on the system implemented by YouTube.

YouTube allows copyright holders to identify when their content has been remixed for use on YouTube videos (their Content ID system). When a match is made, the copyright holder can do various things. It can block content and YouTube will pull the video. It can track the usage of its content. And, finally, it can monetise that content. Under this last option, YouTube shows ads on the content and the copyright holder receives revenue from those ads. At present, YouTube pays out half of the ad revenues to the copyright holder and the performer.

The key feature of this system is that the platform permits users to remix and publish copy protected works. However, it also commits, upfront, to compensation based on a share of revenues accruing to the remixed endeavours. While this is not a remix right per se, it is permissive and encouraging of remixing. What it does, essentially, is ask copyright holders to 'opt out' of revenue sharing and, in the process, potentially block the remixed work. Consequently, it is useful to compare what this does to remixing and overall efficiency.

To do this, we move the platform to the background and assume it does not take a cut itself but merely serves as a commitment mechanism for upfront revenue sharing. In the context of the model here, it is as if $A$ could announce and pre-commit to a revenue share, $r$, of $u(x, y, s)$ that it will require from $B .{ }^{5}$ For the moment, it will be assumed that this transfer takes place if $B$ chooses $s>0$ and that if $B$ does not agree to this, the remix is blocked.

With this mechanism, $A$ and $B$ 's problems become:

$$
\begin{gathered}
x^{*} \in \arg \max _{x} \pi\left(x, s^{*}(x)\right)+r u\left(x, y^{*}(x), s^{*}(x)\right)-C(x) \\
\left\{y^{*}\left(x^{*}\right), s^{*}\left(x^{*}\right)\right\} \in \arg \max _{y, s}(1-r) u\left(x^{*}, y, s\right)-C(y)
\end{gathered}
$$

From this, it is clear that $s_{R C}=1$ as, ex post, $B$ has no incentive to restrict usage. Thus, $B$ chooses $y$ on this basis where $y_{R C} \leq y_{R R}$ as the latter is determined without a share of $u$ appropriated by $A$. For $A$, it has an incentive to increase $u$ as it receives a share of that and unlike under CP, this is not

\footnotetext{
5 YouTube used to only pay a share of revenue to the owner of copy-protected material in a video with the remixer being unable to earn revenue. However, recently, YouTube has relaxed this approach — for instance, for cover songs — and has moved towards a model closer to the one that is specified here.
} 
diminished by $B$ 's bargaining power. Thus, whether $A$ 's choice of $x$ is higher or not depends on comparing $r$ to $\alpha$.

As has been the case throughout this paper, it is instructive to examine the outcomes under the specific functional form assumptions. Here, $y_{R C}=(1-r) \theta x$, while, taking this into account, $x_{R C}=\frac{\beta}{1-2(1-r) r \theta^{2}}$. Given this, it is easy to demonstrate that $A$ 's ex ante expected payoff, $r \theta y_{R C}$ and $x_{R C}$ are all maximized if $r=\frac{1}{2}$. Thus, I will use this as a benchmark for comparison although it should be noted that $B$, if facing an unconstrained choice, would choose a lower $r$. Thus, $x_{R C}=\frac{\beta}{1-\frac{1}{2} \theta^{2}}$ and $y_{R C}=\frac{\theta \beta}{2-\theta^{2}}$.

This set-up does not lead to an easy comparison between copyright regimes. For instance, note that:

$$
x_{C P}>x_{R C} \Rightarrow \frac{1+\beta-\alpha}{1-2 \alpha(1-\alpha) \theta^{2}}>\frac{\beta}{1-\frac{1}{2} \theta^{2}} \Rightarrow \frac{1-\frac{1}{2} \theta^{2}}{\beta \theta^{2}}>\frac{\frac{1}{2}-2 \alpha(1-\alpha)}{1-\alpha}
$$

so long as $\theta>\theta_{C P}$. Otherwise,

$$
x_{C P}>x_{R C} \Rightarrow 1+\beta>\frac{\beta}{1-\frac{1}{2} \theta^{2}} \Rightarrow 1-\frac{1}{2} \theta^{2}>\frac{\beta}{1+\beta}
$$

This occurs because remixing always occurs under RC but only sometimes under CP (and RR). While a high $\alpha$ decreases incentives in remixing and overall profits, under $\mathrm{CP}$, in this case, no remixing will be permitted for precisely that reason.

This suggests that perhaps an alternative (softer) version of RC is warranted. Suppose that ex ante, $A$ nominates a share $r$ if sampling takes place but can also choose not to make that offer and block remixing as a default. Thus, $A$ commits ex ante whether to allow remixing or not in contrast to $\mathrm{CP}$ where it is a negotiation or RR where it is B's discretion. If remixing occurs, this leads to the same outcomes as above where $A$ expects a payoff of $\frac{\beta^{2}}{\left(2-\theta^{2}\right)^{2}} \theta^{2}$. However, if remixing is not permitted, $A$ 's profits are: $\frac{1}{2}(1+\beta)^{2}$. Thus, $A$ will choose to permit remixing if $\frac{\theta}{\left(2-\theta^{2}\right)} \geq \frac{1}{\sqrt{2}} \frac{1+\beta}{\beta}$. This suggests that for high levels of $\beta$ and $\theta$ this regime will encourage more remixing than what would arise under $\mathrm{CP}$; precisely because in cases where such remixing benefits $A, A$ can commit for it to occur.

\section{Conclusion}

This paper has provided the first characterisation of copyright regimes in a framework where copy-protected material can be used for remixed content creation and where negotiations over the 
extent of such activity take place using a property rights approach. In so doing, new rationales in support of traditional copyright protection regimes (where rights reside with the original content creator) have been provided. However, it has also been demonstrated how fair use regimes can improve social welfare and how remix rights regimes can do this as well as minimising transaction costs, if any, associated with negotiation of content reuse.

Empirically, the impact of digitisation provides an opportunity to test the broad theory proposed here. Specifically, digitisation increases the incentives for remixing and consequently the reuse of content in a way that may harm the original content provider's incentives. However, the opportunity to negotiate over the extent of that harm provides a Coasian-route to improved efficiency. Thus, while no copyright protection may lead to worse outcomes for original content provider incentives as digitisation progress, traditional copyright protection may improve the opportunities for remixing as there are more rents to be shared with those original content creators. Interestingly, traditional copyright protection may fail precisely where the returns to remixing afford by digitisation are least. In that situation, a remix rights regime that is more permissive of remixing without negotiation may prove more efficient and lead to higher incentives to create content by both original content creators and remixers. Of course, the extent of that improvement is obviated by the share of original content creator rents that may be dissipated by remixing. 
Figure 1: Timeline

\begin{tabular}{lll}
$\begin{array}{l}\text { Rights } \\
\text { regime } \\
\text { set }\end{array}$ & $\begin{array}{c}A \text { and } B \\
\text { negotiate } \\
\operatorname{over}_{j}(s, t)\end{array}$ & $\begin{array}{c}\text { Payoffs } \\
\text { realised }\end{array}$ \\
\hline \multicolumn{2}{c}{$A$ chooses $x$} & $B$ chooses $y$
\end{tabular}

Figure 2: Optimality of Remix Rights

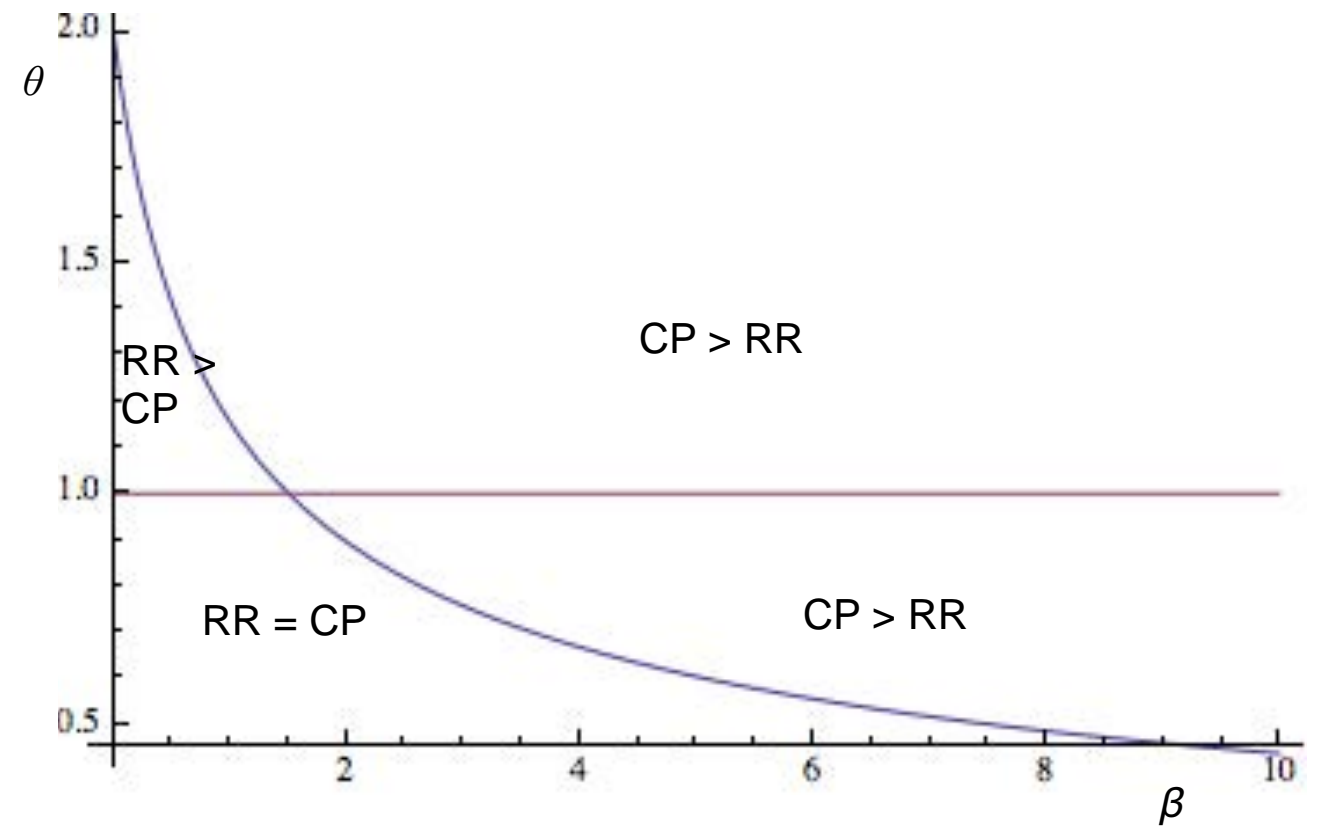




\section{VII.Appendix}

\section{Proof of Proposition 2}

Note first that:

$$
\theta y_{N C}=\frac{(\beta+\alpha(1-s))(s(1-\alpha)+\alpha) \theta^{2}}{1+2(1-s)(s(1-\alpha)+\alpha) \alpha \theta^{2}}<1 \Rightarrow(\beta+(\alpha-2)(1-s))(s(1-\alpha)+\alpha) \theta^{2}<1 .
$$

Consider first an equilibrium where $s_{N C}=0$. In this case, $x_{N C}=\frac{\beta+\alpha}{1+2 \alpha^{2} \theta^{2}}$ and $y_{N C}=\frac{(\beta+\alpha) \alpha \theta}{1+2 \alpha^{2} \theta^{2}}$. A necessary condition for this to be an equilibrium outcome is $(\beta+\alpha-2) \alpha \theta^{2}<1$. This clearly holds if $\beta \leq 2-\alpha$. Otherwise, the equilibrium will require that $\theta^{2}<\frac{1}{(\beta+\alpha-2) \alpha}$.

Now consider a possible equilibrium where $s_{N C}=1$. In this case, $x_{N C}=\beta$ and $y_{N C}=\theta \beta$. A necessary condition for this to be an equilibrium is $\theta^{2} \geq \frac{1}{\beta}$.

To examine whether these are equilibrium outcomes. We consider two cases depending upon whether $\beta \leq(>) 2-\alpha$. First, if $\beta \leq 2-\alpha$, then $\frac{1}{\beta}>\frac{1}{(\beta+\alpha-2) \alpha}$ and, as noted above, $s_{N C}=0$ is an equilibrium outcome. But what happens if $\theta^{2}>\frac{1}{\beta}>\frac{1}{(\beta+\alpha-2) \alpha}$ ? Then $A$ by choosing $x_{N C}=\frac{\beta+\alpha}{1+2 \alpha^{2} \theta^{2}}$ or $x_{N C}=\beta$ can determine whether the negotiated s equals 0 or 1 . A's payoff for a choice of $\mathrm{s}=0$ is $\frac{\beta+\alpha}{1+2 \alpha^{2} \theta^{2}} \beta+\alpha\left(\frac{\beta+\alpha}{1+2 \alpha^{2} \theta^{2}}-\theta \frac{(\beta+\alpha) \alpha \theta}{1+2 \alpha^{2} \theta^{2}}\right)-\frac{1}{2}\left(\frac{\beta+\alpha}{1+2 \alpha^{2} \theta^{2}}\right)^{2}$ while its payoff for a choice of $\mathrm{s}=1$ is $\beta-\frac{1}{2} \beta^{2}$. It is easy to show that the former exceeds the latter. Thus, if $\theta^{2}>\frac{1}{\beta}>\frac{1}{(\beta+\alpha-2) \alpha}$, the unique equilibrium outcome involves $s_{N C}=0$.

Second, if $\beta>2-\alpha$, then note that it can never be the case that $\frac{1}{\beta}>\frac{1}{(\beta+\alpha-2) \alpha}$ as this implies that $(\beta+\alpha-2) \alpha>\beta \Rightarrow(\alpha-2) \alpha>\beta(1-\alpha)$ which can never hold as the LHS is negative. Thus, $\frac{1}{(\beta+\alpha-2) \alpha} \geq \frac{1}{\beta}$. Thus, either (i) $\theta^{2}>\frac{1}{(\beta+\alpha-2) \alpha} \geq \frac{1}{\beta}$; (ii) $\frac{1}{(\beta+\alpha-2) \alpha}>\theta^{2} \geq \frac{1}{\beta}$ or (iii) $\frac{1}{(\beta+\alpha-2) \alpha}>\frac{1}{\beta}>\theta^{2}$. For case (i), $s_{N C}=1$ is the unique equilibrium outcome. For case (ii) we have already noted that this allows $A$ to choose whether remixing occurs or not and $A$ will find it optimal to choose $x$ so that it does not occur. Finally, for (iii), $s_{N C}=0$ or $s_{N C}=1$ is the unique equilibrium outcome.

Finally, note that $\theta^{2}>\frac{1}{(\beta+\alpha-2) \alpha}$ and $\beta>2-\alpha$ implies $\beta>\frac{1}{\theta^{2} \alpha}+2-\alpha$.

\section{Proof of Proposition 3}

First, consider the negotiated choice of $s$. Observe that:

$$
\begin{aligned}
& \theta y_{C P}=\frac{s(1-\alpha)(1+\beta-s \alpha) \theta^{2}}{1-2 s^{2}(1-\alpha) \alpha \theta^{2}}>1 \\
& \Rightarrow s(1-\alpha)(1+\beta-s \alpha) \theta^{2}>1-2 s^{2}(1-\alpha) \alpha \theta^{2} \\
& \Rightarrow \beta>\frac{1}{\theta^{2}(1-\alpha)}-1-\alpha
\end{aligned}
$$

Thus, if $\beta$ is sufficiently high (specifically, $\beta>\frac{1}{\theta^{2}(1-\alpha)}-1-\alpha$ ), $s_{C P}=1$. In this case, $x_{C P}=\frac{1+\beta-\alpha}{1-2 \alpha(1-\alpha) \theta^{2}}$ and $y_{C P}=\frac{\theta(1-\alpha)(1+\beta-\alpha)}{1-2 \alpha(1-\alpha) \theta^{2}}$. Otherwise, $s_{C P}=0$ with $x_{C P}=1+\beta$ and $y_{C P}=0$. 
Comparing these to the equilibrium choices under $\mathrm{NC}$, it is easy to demonstrate that $x_{C P}>x_{N C}$ and that if $\beta \geq \beta_{C P}$, then $y_{C P}>y_{N C}$ as well.

\section{Proof of Proposition 4}

First, note that if the negotiated $s$ is 0 , then surplus is:

$$
\pi\left(x^{*}(0), 0\right)+u\left(x^{*}(0), y^{*}\left(x^{*}(0)\right), 0\right)-C\left(x^{*}(0)\right)-C\left(y^{*}\left(x^{*}(0)\right)\right)=\frac{(1+\beta-\underline{s}(1-\alpha))\left(1+\beta+\underline{s}(1-\alpha)+\underline{s}^{2} \alpha^{2}(3+3 \beta+\underline{s}(1-\alpha)) \theta^{2}\right)}{2\left(1+2 \underline{s}^{2} \alpha^{2} \theta^{2}\right)^{2}}
$$

which is decreasing in $\underline{s}$. Thus, in this case, it is optimal to set $\underline{s}=0$ with resulting surplus of $\frac{1}{2}(1+\beta)^{2}$. On the other hand, if the negotiated $s$ is 1 , then surplus is:

$\pi\left(x^{*}(1), 1\right)+u\left(x^{*}(1), y^{*}\left(x^{*}(1)\right), 1\right)-C\left(x^{*}(1)\right)-C\left(y^{*}\left(x^{*}(1)\right)\right)=\frac{(\beta+(1-\underline{s})(1-\alpha))\left(\beta+\beta(1-(1-\underline{s}) \alpha)(1-3(1-\underline{s}) \alpha) \theta^{2}-(1-\underline{s})(1-\alpha)\left(1-\left(1-(1-\underline{s})^{2} \alpha^{2}\right) \theta^{2}\right)\right)}{2\left(1-2(1-\underline{s}) \alpha(1-(1-\underline{s}) \alpha) \theta^{2}\right)^{2}}$ and becomes negative for low $\beta$. If we take the derivative of this with respect to $\underline{s}$ and then look at the limit as $\underline{s}$ goes to 1 , we have $-\beta \theta^{2}\left(1-\alpha+2 \beta \alpha \theta^{2}\right)<0$. Note that, if $s=1$, then the surplus (when $\underline{s}=1$ ) becomes: $\frac{1}{2} \beta^{2}\left(1+\theta^{2}\right)$ which exceeds $\frac{1}{2}(1+\beta)^{2}$ (the surplus when $\underline{s}=0$ ) as $\beta$ becomes large.

\section{Proof of Proposition 5}

In this case, $x_{R R}=\beta+1$ while $y_{R R}=\theta s$. Consequently, $s_{R R}=1$ if $\theta>\theta_{R R}=1$ and 0 otherwise. For comparisons, note first that when $s_{C P}=0$, then $x_{R R}=x_{C P}>x_{N C}$ and $y_{R R}=y_{C P}=0$. Note that for $\mathrm{CP}$, this occurs when $\theta<\theta_{C P}=\frac{1}{\sqrt{(1-\alpha)(\beta+1+\alpha)}}$ where $\theta_{R R}>\theta_{C P} \Rightarrow 1>\frac{1}{\sqrt{(1-\alpha)(\beta+1+\alpha)}} \Rightarrow \beta>\frac{\alpha^{2}}{1-\alpha}$.

When $\theta \geq \theta_{R R}, \theta_{C P}, s_{R R}=s_{C P}=1$ and it can be seen that:

$$
x_{C P}>x_{R R} \Rightarrow x_{C P}=\frac{1+\beta-\alpha}{1-2 \alpha(1-\alpha) \theta^{2}}>1+\beta \Rightarrow \theta>\frac{1}{\sqrt{(1+\beta) 2(1-\alpha)}}
$$

Note that $\theta_{C P}>\frac{1}{\sqrt{(1+\beta) 2(1-\alpha)}}$, and thus, this relationship holds. By contrast,

$$
y_{C P}>y_{R R} \Rightarrow \frac{\theta(1-\alpha)(1+\beta-\alpha)}{1-2 \alpha(1-\alpha) \theta^{2}}>\theta \Rightarrow \theta^{2}>\frac{-\beta+\alpha \beta-\alpha^{2}}{2 \alpha(1-\alpha)}
$$

which always holds.

When $\theta_{R R}>\theta>\theta_{C P}$ (which can arise if $\beta>\frac{\alpha^{2}}{1-\alpha}$ ), $s_{R R}=0$ while $s_{C P}=1$ and it is clear that both $x_{C P}>x_{R R}$ and $y_{C P}>y_{R R}$.

Finally, if $\theta_{C P}>\theta>\theta_{R R}$ (which can arise if $\beta<\frac{\alpha^{2}}{1-\alpha}$ ), $s_{R R}=1$ while $s_{C P}=0$. In this case, $x_{R R}=x_{C P}>x_{N C}$ while $y_{R R}>y_{C P}=0$. 


\section{References}

Boldrin, M. and D.K. Levine (2010), Against Intellectual Monopoly, Cambridge University Press: Cambridge.

Depoorter, B., \& Parisi, F. (2002). Fair use and copyright protection: a price theory explanation. International Review of Law and Economics, 21(4), 453-473.

Green, J. and Scotchmer (1995), On the division of profit in sequential innovation. RAND Journal of Economics, 26 (1), 20-33.

Hart., O.D. (1995), Firms, Contracts and Financial Structure, Oxford University Press: Oxford.

Halonen-Akatwijuka, M. and T. Regner (2009), Digital Technology and the Allocation of Ownership in the Music Industry. Jena Economic Research Paper, No.96, Max Planck.

Johnson, W. R. (1985). The Economics of Copying. Journal of Political Economy, 93(1), 158-174.

Katz, A. (2013). Copyright and competition policy, in R. Towse and C. Handke (eds.), Handbook of the Digital Creative Economy, Edward Elgar: Cheltenham, 209-21.

Landes, W. M., \& Posner, R. A. (1989). An economic analysis of copyright law. The Journal of Legal Studies, 18(2), 325-363.

Lessig, L. (2008), Remix: Making Art and Commerce Thrive in the Hybrid Economy, Penguin: New York.

Miceli, T. J., \& Adelstein, R. P. (2006). An economic model of fair use. Information Economics and Policy, 18(4), 359-373.

Novos, I. E., \& Waldman, M. (1984). The Effects of Increased Copyright Protection: An Analytic Approach. Journal of Political Economy, 92(2), 236-246.

Pitchford, R., \& Snyder, C. M. (2003). Coming to the Nuisance: An Economic Analysis from an Incomplete Contracts Perspective. Journal of Law, Economics, and Organization, 19(2), 491516. 